\title{
Road Side Unit Assisted Stochastic Multi-hop Broadcast Scheme for Instant Emergency Message Propagation
}

\author{
Xing Fan*, Bo Yang*, Ryo Yamamoto*, Yoshiaki Tanaka*,** \\ * Global Information and Telecommunication Institute, Waseda University \\ 1-3-10 Nishi-Waseda, Shinjuku-ku, Tokyo, 169-0051 Japan \\ ** Research Institute for Science and Engineering, Waseda University \\ 17 Kikuicho, Shinjuku-ku, Tokyo, 162-0044 Japan
}

fan.xing@asagi.waseda.jp, yangbo_youhaku@ruri.waseda.jp, ryo_yamamoto@moegi.waseda.jp, ytanaka@waseda.jp

\begin{abstract}
VANET (Vehicular ad hoc Network) is a special kind of ad hoc wireless network where every single node is a vehicle moving in a relatively high velocity, which leads to exclusive challenges like rapid changing topologies, safety and privacy concerns. In this specific network, the propagation of emergency messages could be critical to save human lives and property. Many researchers have proposed routing or broadcast protocols to solve the problems in VANET. The objective of this paper is to propose a broadcast scheme in VANET that is not likely to cause broadcast storm problem with a reasonable delay and high delivery rate. Since VANET is an attack-prone network and any kind of malicious behaviour in VANET might cause serious loss or even death in reality, we should also refrain from using beacons to exchange privacy-sensitive information in $\mathrm{V} 2 \mathrm{~V}$ (Vehicle to Vehicle). In this paper, a multi-hop broadcast scheme that makes use of RSU and V2I (Vehicle to Infrastructure) communication is proposed. The simulation result shows that the proposed scheme outperforms static stochastic broadcast scheme in terms of delivery rate. Comparing to flooding, we offer a better delay and less network usage.
\end{abstract}

Keywords — VANET, Broadcast, V2I, DSRC, Percolation, Stochastic

\section{INTRODUCTION}

Wireless ad hoc network is a network that does not rely on existing infrastructure to relay packets and uses all nodes as clients and routers. In this way, it does not require any type of access points or routers to be pre-installed in the network. It is decentralized, dynamic and easy-to-deploy characteristics make this kind of network a very active research area.

One of the most promising and practical topics is vehicular ad hoc network (VANET) [1]. Research in this area is significant because the increasing car accident and safety related problems [2] are threatening human lives and property, and intelligent transportation system (ITS) is designed to help solving these problems. In developing countries, motorization is happening in urban area with high population and cars are moving at high rather speed in these regions, which is considerably dangerous. Vehicle manufactures are developing various ITS systems to assist and protect driver's life and their property. VANET is one of the most basic systems to be implemented.

VANET is formed by vehicles, which allows nodes to perform vehicle to vehicle (V2V) and vehicle to infrastructure (V2I) communication to obtain traffic condition, alert messages and benefit from navigation system. Its specialty also gives the following unique characteristic to VANET:

- Nodes move at rather high velocity

- Nodes move in an organized, predictable way

- Nodes are assumed to have modern on-board-unit (OBU) installed, including GPS, WIFI connectivity, etc.

- A special kind of stationary nodes could exist, which is called road-side-unit (RSU), as is shown in Figure 1

The previous listed characteristics makes every applicable protocol and scheme in VANET requires much more detailed design rather than directly import from existing ad hoc network technique such as mobile ad hoc network(MANET) [3]. In addition to other ad hoc network, researchers need to take the following risks into consideration in VANET:

- The usage of privacy-sensitive information

- Easily fragmented network topology

- Security

- Scalability

Since most of concerns in VANET are safety of the vehicles and their drivers, it is not difficult to infer that emergency broadcast is one of the most critical areas in VANET communication. Every car accident, natural disaster and other alerts need to be propagated in the network very fast with reliability. Optimization of emergency message propagating includes many areas such as reliability research, broadcast schemes in VANET and privacy protection. In these areas, broadcast schemes are the most direct contributors. For this reason, this paper mainly focused on emergency broadcast schemes in VANET. As a common methodology in propagating emergency messages, broadcast is widely used in many areas. Pure flooding is one of the most simple and effective methods, namely every node must retransmit the packet when it receives the one. Although this scheme is good enough for simple situation, it is not robust to be applied for practical usage. This is because flooding packets blindly 


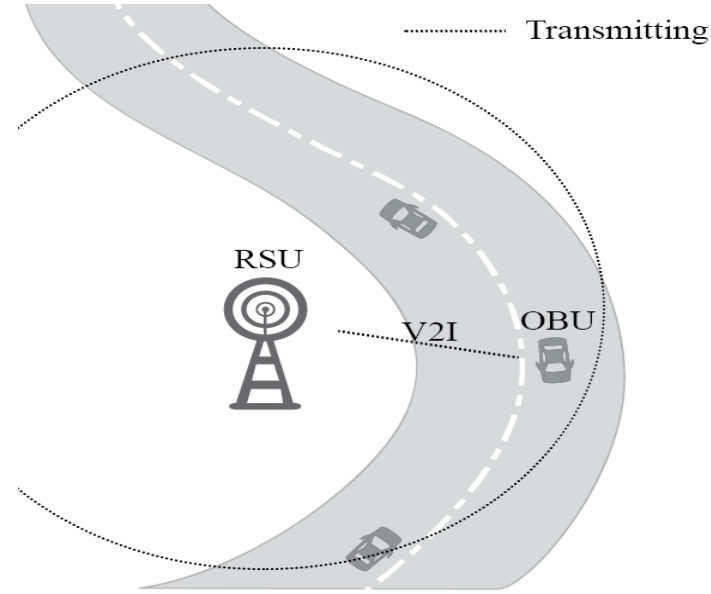

Figure 1. RSU and V2I in VANET

will cost too many resources in the network and finally lead to collisions or even unresponsiveness (broadcast storm problem).

In order to solve this problem, many approaches were proposed [4]. In counter-based approaches, number of rebroadcasting is limited and nodes would not retransmit a packet after the packet reaches a certain counter. In locationbased approaches, only nodes in a specified location retransmit a packet. In distance-based approaches, only nodes with furthest distance to a source node can retransmit a packet. In cluster-based approaches, packet retransmitting relies on certain nodes within a specified structure. In probabilitybased approaches, every node has a possibility to retransmit packet when they receive one.

Each solution proposed above has its pros and cons. Counter-based approaches are prone to malicious modification since the counters are usually stored in the message. Location-based approaches specified location area in packets. Thus, it is also prone to attacks since attackers can easily obtain information of broadcasting area. Distance-based approaches are the state of the art broadcast scheme because it tends to select the furthest nodes as relays. Thus, it maximizes the dissemination ranges. On the other hand, it does not have any control scheme once a packet is broadcasted to networks. Probability-based approaches are flexible schemes since all their behaviours rely on how to decide the probability, depending on different scenarios researchers use different approaches to adjust the rebroadcast probability accordingly.

The effectiveness of broadcast also heavily depends on a technique of packet transmission. Dedicated short-range communication (DSRC) [5] allows high-speed connection in both $\mathrm{V} 2 \mathrm{~V}$ and V2I. It uses $5.9 \mathrm{GHz}$ band and transmission range could reach $1 \mathrm{~km}$. This allows us to transmit packet over 3 times further than conventional IEEE 802.11 [6] and also mitigates a fragmentation of network.

As is mentioned previously, there are special class of nodes called RSU exist exclusively in VANET. These nodes are usually installed in an intersect area on a highway, gas station and the other area according to the node density. These RSUs are rarely used in conventional VANET broadcast.

This paper is organized as follows: introduction of some existing broadcast schemes are in Section II, the proposed network architecture and usage of RSU are explained in Section III, detailed design of broadcast scheme is written in Section IV, simulation and result are recorded in Section V, Section VI conclude this paper.

\section{RELATED WORKS}

Most of the practical broadcast schemes are either variation or hybrid of the 4 basic broadcasts: Counter, Location, Distance and Probability [4].

Smart broadcast [7] is a variation of distance-based broadcast, the area around a source node is divided into different sections as shown in Figure 2, which allows every node to have their own contention window. In this way, the outer most nodes in S2 have the highest priority to access the channel. This scheme provides a way to prioritize a rebroadcast using distance-based broadcasting scheme and it is practical and it guarantees maximum dissemination range.

Another interesting broadcasting is called stochastic broadcast for VANET [8], which is a combination of distancebased broadcast and stochastic broadcast. The paper proposed two ways to determine a retransmit probability: one is fixed; another is according to a one-hop distance. That research has following 2 advantages. First, every node determines a probability by itself, which means transmission is not used except broadcasting. This eliminates a privacy issues and provides scalability. Second, it uses a percolation study to estimate a theoretically maximum connectivity. However, this paper does not mention about dynamical adjustment of the probability according to a network condition changes. In this paper, the proposed scheme utilizes RSU to deal with the rapid fragmented network.

The objective of this paper is to propose a broadcast that could propagate emergency messages with high deliver rate, reasonable delay and does not overwhelm a network with broadcast storm. Privacy is also taken into consideration by limit a usage of beacons.

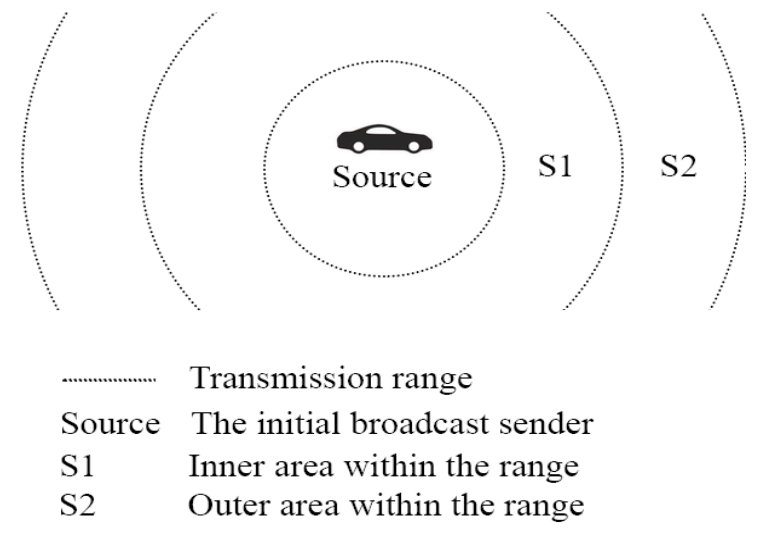

Figure 2. Sections in smart broadcast

\section{NETWORK ARCHITECTURE}

VANET could be simplified into two scenarios: city with high node density, highway with sparse density and easily fragmented. Oversimplify the VANET could lead to assume 
the network is connected, which ignores the fact that VANET is easily fragmented due to its nature [9]. In this paper, instead of making such assumptions, we use RSU to mitigate network fragmentation and use percolation to help us study fragmentation.

To deal with fragmentation problems in VANET, this paper proposes a usage of RSU to assist a broadcast. This paper assumes that every node equips an OBU with DSRC and GPS enabled. Moreover, limited number of RSUs was installed along the road. Although how to deploy RSUs is out the scope this paper, we assume a generic situation. That is, RSUs are deployed in a scattered manner that cannot form a connected network and every RSU works independently.

In a multi-hop broadcast, when a source node initializes a broadcast, it simply broadcasts a message and utilizes some mechanism to let other nodes relay it. In conventional broadcast schemes, there are no roles for RSU to participate in this process. Theoretically, putting RSUs into broadcast process by letting them rebroadcast with magnified signal and further transmitting range is beneficial. Here, we proposed a mechanism to be installed on RSUs that could still work independently to participate and contribute to the broadcast process even if they are not connected.

The proposed scheme for RSU in VANET broadcast could be divided into following two parts. For the first part, RSU should use a maximum transmission range to rebroadcast a message when it receives the one. For the second part, to mitigate the broadcast storm, it should suppress a further rebroadcast in the inner region of its broadcast range by using certain approach as shown in Figure 3. The detailed broadcast scheme for RSU side is described as follows:

(1) When RSU receives a broadcast message, check if it is duplicate message.

(2) If it is not duplicated, RSU immediately preform a rebroadcast burst with a message that contains the original message and also two addition scalar values: broadcast suppression range $R_{S}$ and minimum broadcast suppression $P_{m}$.

Note that $R_{s}$ and $P_{m}$ are used to suppress the rebroadcast of nodes in the transmitting range of RSU.
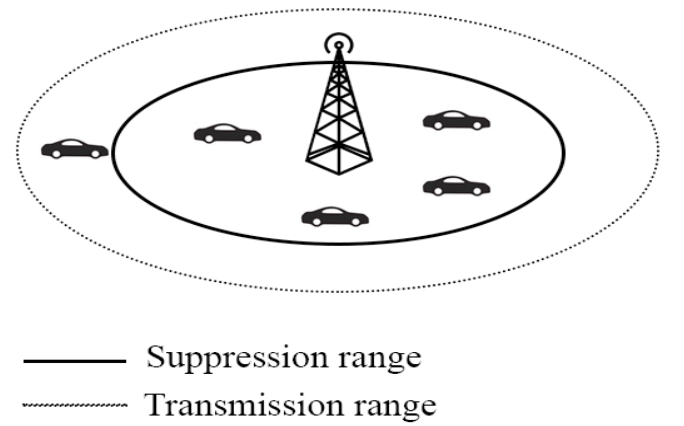

The rebroadcast within the suppression range will be reduces.

Figure 3. RSU broadcast suppression

\section{IV.Proposed BRoAdCAST SCHEME}

The proposed broadcast scheme was developed on top of the basic probability-based broadcast, which is explained as follows:

(1) Every node maintains a value $P_{c}$, which indicates the current rebroadcast probability.

(2) While receiving a non-duplicate broadcast message, a node enters a probability check process that would cause the node has the probability $P_{c}$ to rebroadcast the message after a default contention window.

(3) After a probability check, no matter the node retransmits the message or not, $P_{c}$ is reset to a default value $P_{d}$.

Note that $P_{c}$ was provided depending on different broadcast schemes, which will be discarded every probability check. $P_{d}$ is the default broadcast probability, which enables every node to have $P_{d}$ even if $P_{c}$ was not set.

To align the behaviour of OBUs to the proposed scheme, this paper proposes the following scheme for OBU to deal with broadcasts from RSU:

(1) When an OBU receives a broadcast message from RSU, it checks the message whether it is a duplicate message or not.

(2) If it is not duplicated, OBU uses either signal strength or location services to get its distance to the corresponding RSU, which is denoted as $R_{r o}$.

(3) If $R_{r o}<$ broadcast suppression range $R_{s}$, set the current rebroadcast probability to suppression minimum $P_{m}$.

(4) Proceed to probability check.

Considering the broadcast burst from RSU is likely to cover more area with stronger signal strength, the proposed scheme utilizes the scheme mentioned above to ensure that broadcasts from RSU suppress other broadcast within the radius of $R_{s}$ to suppression minimum value $P_{m}$.

Although this paper focused on using V2I to maximum the effectiveness of the broadcast, it is imperative to keep in mind that $\mathrm{V} 2 \mathrm{~V}$ is still doing a heavy lifting in the broadcast propagating process. Therefore, this paper proposes a dynamic scheme for $\mathrm{V} 2 \mathrm{~V}$ communication, which is backed by percolation study.

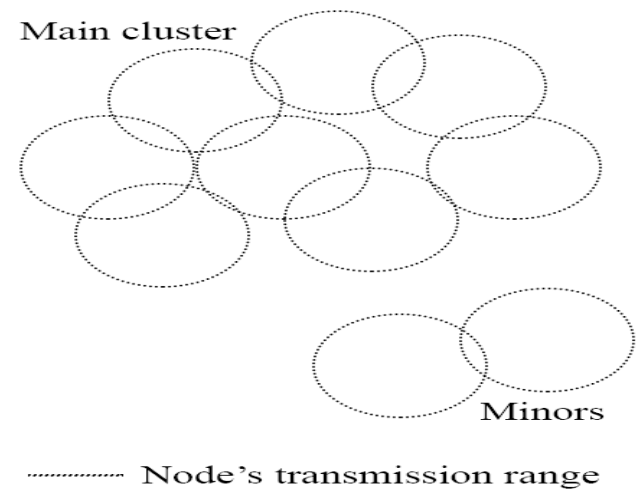

If any minor clusers exist, the network is fragmented.

Figure 4. Percolation 
Wireless ad hoc network could be simplified into a model that consists of infinite nodes generated by a Poisson process using certain density. With the density increased, the chance of all the nodes forming a connected graph is also increased. Continuum percolation is a study that dedicates to analyse this model [10].

If nodes are distributed in a space by density $\rho$, we could denote an expected neighbourhood of a certain node as $\lambda$ is calculated using following Eq.(1) [11]:

$$
\lambda=\rho \pi r^{2}
$$

where $r$ denotes a node transmission range. With higher $\lambda$, it is more likely that infinite nodes are in one connected cluster as is shown in Figure 4. The author of [8] used $\lambda_{c}=4.508$ to 4.515 and verified this value to have $99.99 \%$ probability to form one connected cluster, which means no minors such as shown in Figure 4. In this paper, we try to align the proposed scheme to match this value in order to maximize the broadcast effectiveness.

As is explained previously, if a node does not participate rebroadcasting in the broadcast process, the node is considered to be not in this network [8]. In stochastic broadcast, we have Eq.(2):

$$
\lambda_{s}=P \rho \pi r^{2}
$$

where $P$ denotes the rebroadcast probability. For example, if $\lambda=10$ and the rebroadcast probability for every node is $50 \%$, $\lambda_{s}=5$ is good enough to ensure the network to be connected, that is, the broadcast can be propagated. As explain in continuum percolation, please note that the ideal situation is $\lambda_{s}=4.5$, which means rebroadcast probability should be roughly $45 \%$.

In order to maintain $\lambda_{s}$, it is necessary to adjust $P$ dynamically while the network topology changes. Thus, every node needs to maintain a neighbour node list using either beacons or location services. In this paper, we assume every node maintains a neighbour list using a simple one-hop beacon and total neighbourhood count to be $N$.

The V2V communication scheme is explained as follows:

- Once the node receives a non-duplicate broadcast message from another vehicle, set $P_{c}$ using the following Eq.(3):

$$
P_{c}= \begin{cases}0 & (N=0) \\ 1 & (0<N<5) \\ \frac{4.5}{N} & (N \geq 5)\end{cases}
$$

(1) Reset the neighbour list.

(2) Proceed to probability check.

(3) Reset $P_{c}$ to $P_{d}$

In this way, $P_{c}$ could be aligned to a reasonable value using $N$. Since $N$ is a dynamic value and constantly changing, $P_{c}$ is theoretically always an optimal value.

\section{Performance evaluation}

To evaluate the performance of the proposed scheme, a simulation was conducted in two different scenarios using different sets of parameters. In this simulation, static stochastic broadcast scheme, pure flooding scheme and the proposed is evaluated in both city and highway environment. General parameter used in this simulation is listed in Table 1.

In the simulation, the performance of a broadcast scheme was evaluated using three metrics: message delivery rate, network delay and number of broadcasted packets.

Message delivery rate is the basic performance evaluation, which indicates if the message was successfully delivered to all nodes. Network delay basically shows how long it takes for the message to propagate to all possible nodes and it evaluates the effectiveness of each rebroadcast. As message travels along many hops, the delay increases. Number of broadcasted packets indicates how many broadcast packets are used in the broadcast process, it evaluates the network usage and potential broadcast storm problems.

Since pure flooding rebroadcast every message it receives, it can achieve the theoretically highest deliver rate and generate the most packets.

As shown in Table 2, the parameters used in the first simulation aim to recreate a broadcast on highway scenario with the aid of RSUs installed along the road. The result was shown in Figures 5-7.

TABLE 1. General Stochasic BroadCast SimUlation Parameters

\begin{tabular}{c|l}
\hline Parameter & \multicolumn{1}{|c}{ Value } \\
\hline$R_{s}$ & $0.8 \times \mathrm{RSU}$ transmission range \\
\hline$P_{m}$ & 0.05 \\
\hline$P_{d}$ & 0.2 \\
\hline $\begin{array}{c}\text { Communication } \\
\text { range V2V }\end{array}$ & $1 \mathrm{~km}$ \\
\hline RSU burst & 3 times without contention window \\
\hline RSU burst range & $1.5 \mathrm{~km}$ \\
\hline $\begin{array}{c}\text { Beacon } \\
\text { contention }\end{array}$ & $\leq 1 \mathrm{~ms}$ \\
\hline $\begin{array}{c}\text { Rebroadcast } \\
\text { contention }\end{array}$ & $\leq 2 \mathrm{~ms}$ \\
\hline
\end{tabular}

TABLE 2. Simulation 1 Parameters

\begin{tabular}{c|l}
\hline Parameter & \multicolumn{1}{|c}{ Value } \\
\hline Area & $20 \mathrm{~km} \times 0.1 \mathrm{~km}$ \\
\hline $\begin{array}{c}\text { Number of } \\
\text { RSU }\end{array}$ & 5 \\
\hline $\begin{array}{c}\text { RSU } \\
\text { distribution }\end{array}$ & Uniform, installed along the road \\
\hline Mobility & Random waypoint \\
\hline Speed & $50 \mathrm{~km} / \mathrm{h}$ to $80 \mathrm{~km} / \mathrm{h}$ \\
\hline $\begin{array}{c}\text { Number of } \\
\text { nodes }\end{array}$ & $\begin{array}{l}15 \text { to } 45(10 \text { to } 40 \text { for RSU-Assisted } \\
\text { broadcast })\end{array}$ \\
\hline
\end{tabular}




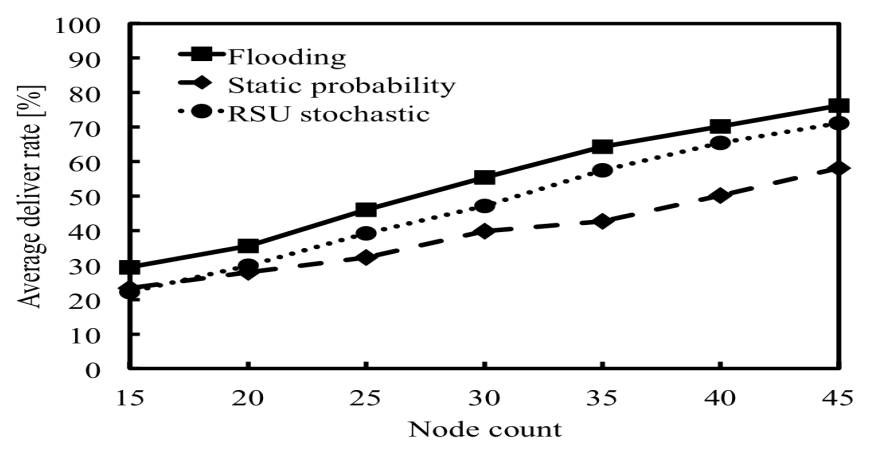

Figure 5. Message deliver rate in highway simulation scenario

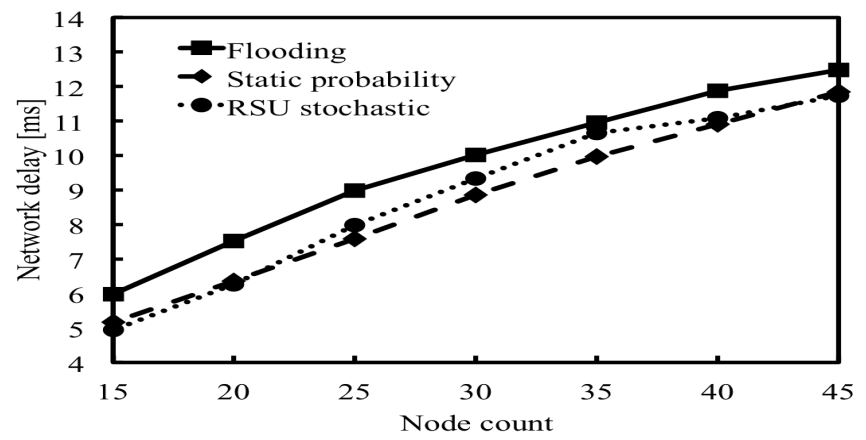

Figure 6. Delay in highway simulation scenario

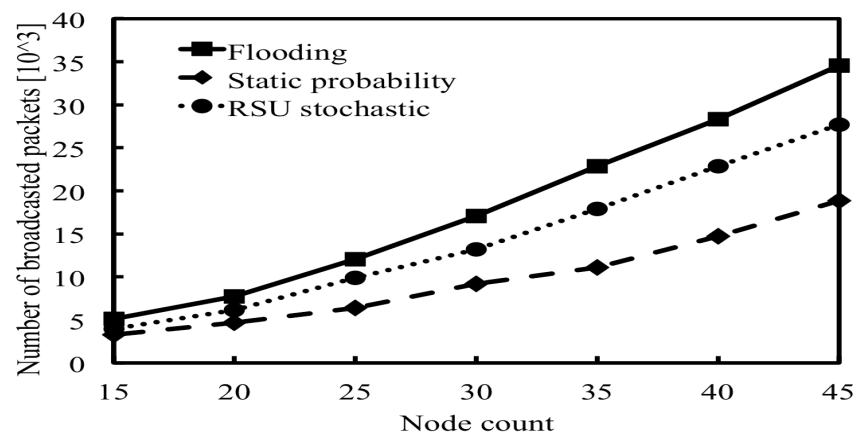

Figure 7. Packet usage in highway simulation scenario

From Figures 5 and 6 , the proposed broadcast scheme achieved better deliver rate and lower delay at the same time in high node density due to higher RSU's coverage. Since deliver rate has been increased, number of broadcasted packets will increase in order to cover more nodes. In Figure 7 , we can see that number of broadcasted packets is increasing but please note there is an overhead cause by proposed method.

As shown in Table 3, the parameters we used in the next simulation are about recreate a broadcast in city scenario where RSUs are randomly installed in the city.

In city scenario the proposed broadcast scheme got a performance boost in the sense of delay and packet usage due to the sparse distributed RSUs comparing to highway scenario. From Figures 8 and 9 the proposed broadcast scheme achieved higher deliver rate with almost the same delay comparing with static stochastic broadcast. If comparing Figure 10 with
Figure 7, it is obvious that in city scenario packet usage also decreased. Achieving high deliver rate with lower delay and lower packet usage means every broadcast covers more nodes, which is our intention to mitigate broadcast storm problem.

TABLE 3. SIMULATION 2 PARAMETERS

\begin{tabular}{c|l}
\hline Parameter & \multicolumn{1}{|c}{ Value } \\
\hline Area & $9 \mathrm{~km} \times 9 \mathrm{~km}$ \\
\hline $\begin{array}{c}\text { Number of } \\
\text { RSU }\end{array}$ & 5 \\
\hline $\begin{array}{c}\text { RSU } \\
\text { distribution }\end{array}$ & Random \\
\hline Mobility & Random waypoint \\
\hline Speed & $10 \mathrm{~km} / \mathrm{h}$ to $30 \mathrm{~km} / \mathrm{h}$ \\
\hline Node count & $\begin{array}{l}15 \text { to } 45(10 \text { to } 40 \text { for RSU-Assisted } \\
\text { broadcast })\end{array}$ \\
\hline
\end{tabular}

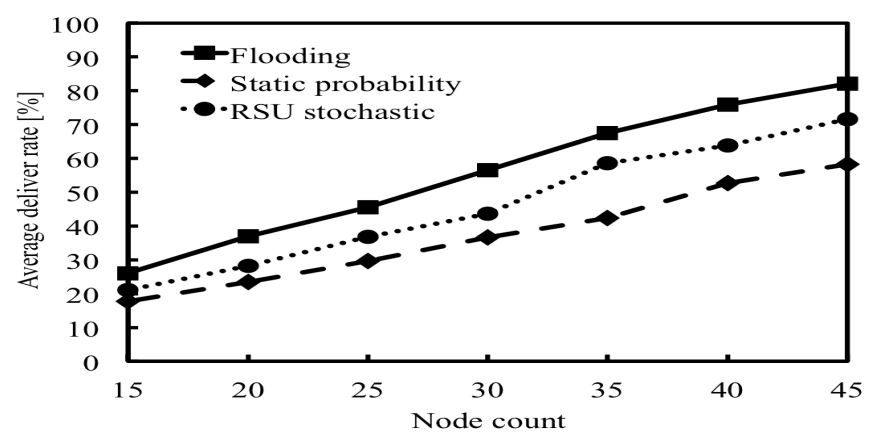

Figure 8. Message deliver rate comparision in city simulation scenario

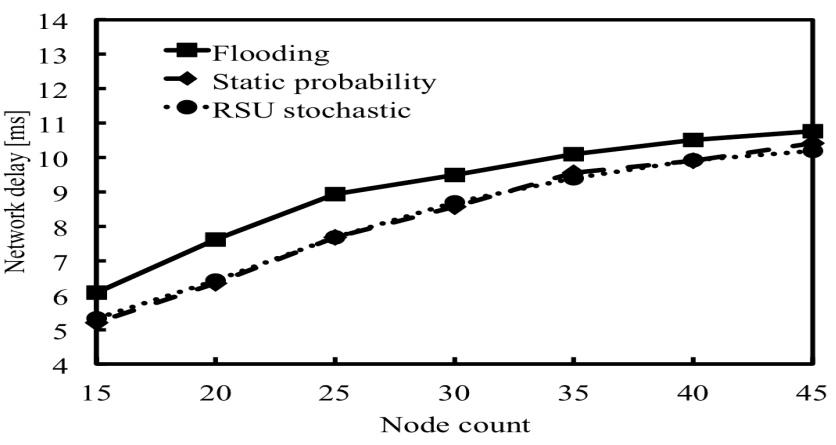

Figure 9. Delay comparision in city simulation scenario

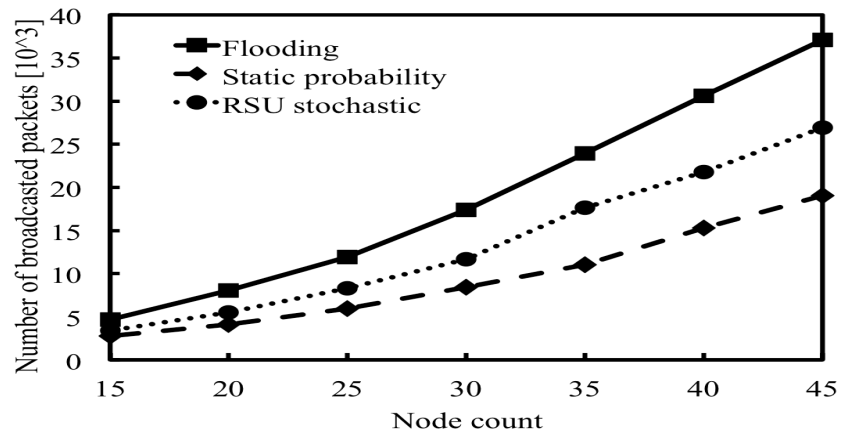

Figure 10. Packet usage comparision in city simulation scenario 
Since RSUs are distributed randomly in city scenario, message deliver rate dropped a little comparing to the highway scenario from Figure 8 and Figure 5. In general, the proposed broadcast scheme outperforms the static stochastic broadcast and went toward the upper bound, without bring heavy traffic to the network and cause broadcast storm problem (comparing to flooding). Also, there's no need to acquire the network condition in order to calculate the broadcast probability before the broadcast (comparing to static stochastic broadcast).

\section{CONCLuSiON}

This paper introduced the challenges in VANET emergency message broadcast and its contribution of protecting human lives and properties. It also summarized our objectives to contribute to emergency message broadcast in VANET research. This paper also categorized different broadcast schemes, analysed their strength and weakness. On top of that, this paper introduced two broadcast schemes that related to the study.

In the discussion of exclusive characteristics of VANET, this paper proposed the usage of RSU to assist to broadcasting process, explained the scheme for RSUs to work actively and individually in the broadcast.

By simplify VANET model using percolation theory, this paper developed another scheme to be used in $\mathrm{V} 2 \mathrm{~V}$ broadcasting. In this discussion, this paper explained how to achieve the theoretically highest performance using stochastic model and apply the result in the proposed schemes.

In order to study the performance of the proposed scheme, this paper conducted two simulations. From the result, the proposed scheme achieves our goals by increasing deliver rate and does not bring huge impact on delay and network usage, especially in city scenario.

The amount and position of installed RSUs may have impact on the performance. Moreover, using beacons is not absolutely the ideal solution. We still have some future works such as evaluating the performance impact of RSUs and finding alternative, safer way to transfer beacons.

\section{REFERENCES}

[1] M. L. Sichitiu; M. Kihl, "Inter-vehicle communication systems: a survey," IEEE Commun. Surveys \& Tutorials, vol. 10, no. 2, pp. 88105, Second Quarter 2008.

[2] Bureau of Transportation Statistics, "National transportation statistics," US Department of Transportation [Online]. Available:

http://www.rita.dot.gov/bts/sites/rita.dot.gov.bts/files/publications/natio nal_transportation_statistics/index.html, Accessed Oct. 2013.

[3] B. Williams and T. Camp. "Comparison of broadcasting techniques for mobile ad hoc networks." 3rd ACM Int. Symp. Mobile Ad Hoc Netw. \& Comput., pp. 194-205, June 2002.

[4] S.-Y. Ni, Y.-C. Tseng, Y.-S. Chen, and J.-P. Sheu, "The broadcas storm problem in a mobile ad hoc network," in 5th annual ACM/IEEE Int. Conf. Mobile Comput. and Netw. pp. 151-162. Aug. 1999.

[5] D. Jiang, V. Taliwal, A. Meier, W. Holfelder, and R. Herrtwich, "Design of $5.9 \mathrm{GHz}$ DSRC-based vehicular safety communication", IEEE Trans. Wireless Communication, vol. 13, no. 5, pp. 36-43, Oct. 2006

[6] X. Ma and X. Chen, "Performance analysis of IEEE 802.11 broadcast scheme in ad hoc wireless LANs," IEEE Trans. Vehicluar Technology, vol. 57 , no. 6 , pp. $3757-3767$, Nov. 2008.
[7] E. Fasolo; A. Zanella, and M. Zorzi, "An effective broadcast scheme for alert message propagation in vehicular ad hoc networks," in 2006 IEEE International Conference on Communications, vol.9, pp. 39603965. June 2006.

[8] M. Slavik and I. Mahgoub, "Stochastic broadcast for VANET," in 7th IEEE Consumer Commun. and Netw. Conf., pp. 1-5. Jan. 2010.

[9] N. Wisitpongphan, F. Bai, P. Mudalige, V. Sadekar, and O. K. Tonguz, "Routing in sparse vehicular ad hoc wireless networks," IEEE J. Sel.Areas Commun., vol. 25, no. 8, pp. 1538-1556, Oct. 2007.

[10] B. Bollobas and O. Riordan, Percolation, Cambridge Univ. Press, 2006.

[11] P. Balister, B. Bollobas, and M. Walters, "Continuum percolation with steps in the square or the disc," Random Struct. Alg., , vol. 26, no. 4, pp. 392-403, April 2005.

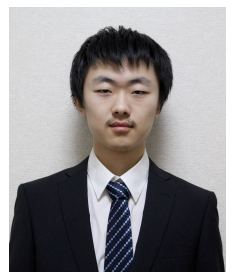

Xing Fan was born in Shanxi, China on June 30th. Xing Fan earned bachelor of engineering in Beijing University of Technology in July 2012. Xing Fan is a master student majored in ad hoc network at Waseda University, Japan while presenting this paper.

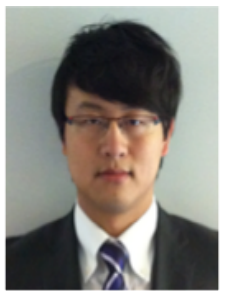

Bo Yang received his B. E. degree in computer science and technology from Xi Dian University, Xi'an, China, in 2009. He received his second M.E. degree in Information Communication from Waseda University, Tokyo, Japan, in 2012. Currently, he is working toward the $\mathrm{Ph}$. D degree in the Global Information and Telecommunication Studies, Waseda University, Tokyo, Japan. He won the ICACT best paper award in Feb. 2012.

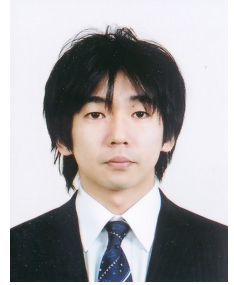

Ryo Yamamoto received his B.E. and M.E. degree in electronic information systems from Shibaura Institute of Technology, Tokyo, Japan, in 2007 and 2009. He received D.S. in global telecommunication studies from Waseda University, Tokyo, Japan, in 2013. He is presently a research associate of Global Information and Telecommunication Institute, Waseda University. He received the IEICE young researcher's award in 2010. His current research interests are mobile ad hoc networks and cross-layered protocols.

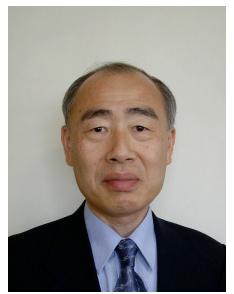

Yoshiaki Tanaka received the B.E., M.E., and D.E. degrees in electrical engineering from the University of Tokyo, Tokyo, Japan, in 1974, 1976, and 1979, respectively. He became a staff at Department of Electrical Engineering, the University of Tokyo, in 1979, and has been engaged in teaching and researching in the fields of telecommunication networks, switching systems, and network security. He was a guest professor at Department of Communication Systems, Lund Institute of Technology, Sweden, from 1986 to 1987 . He was also a visiting researcher at Institute for Posts and Telecommunications Policy, from 1988 to 1991, and at Institute for Monetary and Economic Studies, Bank of Japan, from 1994 to 1996 . He is presently a professor at Global Information and Telecommunication Institute, Waseda University, and a visiting professor at National Institute of Informatics. He received the IEEE Outstanding Student Award in 1977, the Niwa Memorial Prize in 1980, the IEICE Achievement Award in 1980, the Okawa Publication Prize in 1994, the TAF Telecom System Technology Award in 1995 and in 2006, the IEICE Information Network Research Award in 1996, in 2001, in 2004, and in 2006, the IEICE Communications Society Activity Testimonial in 1997 and in 1998, the IEICE Switching System Research Award in 2001, the IEICE Best Paper Award in 2005, the IEICE Network System Research Award in 2006, in 2008, and in 2011, the IEICE Communications Society Activity Award in 2008, the Commendation by Minister for Internal Affairs and Communications in 2009, the APNOMS Best Paper Award in 2009 and in 2012, and the IEICE Distinguished Achievement and Contributions Award in 2013. He is a Fellow of IEICE. 